\title{
Defect Identification of Lumber through Correlation Technique with Statistical Feature Extraction Method
}

\author{
R.Athilakshmi \\ Lecturer, CA Department, \\ Sri Krishna College of Engineering \\ \& Technology, Coimbatore, TN, \\ India
}

\author{
Dr. Amitabh Wahi \\ Professor, IT Department, \\ Bannari Amman Institute of Technology: \\ Sathyamangalam - 638 401, TN, India
}

\author{
Dr. B. Nagarajan \\ Associate Professor, CA Department, \\ Bannari Amman Institute of \\ Technology, Sathyamangalam - 638 \\ 401, TN, India
}

\begin{abstract}
Feature extraction is an important component of a pattern recognition system. A well-defined feature extraction algorithm makes the identification process more effective and efficient. Quality checking is one of the most prominent steps in many applications using Feature extraction. Several techniques exist for the quality checking of wooden materials. However, image based quality checking of wooden materials still remains a challenging task. Although trivial quality checking methods are available, they do not give useful results in most situations. This paper addresses the issue of quality checking of wooden materials using feature extraction techniques with high accuracy and reliability. Experiments conducted under the proposed conditions showing significant results are presented.
\end{abstract}

Keywords — Feature Extraction, Correlation Coefficient, Quality Checking, Defects of Wood.

\section{INTRODUCTION}

The appearance of sawn timber has huge natural variations that the human inspector feels difficult when determining the types of defects and the grade of each board. However, for automatic wood inspection systems these variations are a major source for complication. This makes it difficult to use textbook methodologies for visual inspection. These methodologies generally aim at systems that are trained in a supervised manner with samples of defects and good material, but selecting and labelling the samples is an errorprone process that limits the accuracy that can be achieved.

\section{RELATED WORK}

Quality checking has been a focus of investigation over last decades. Material quality checking plays a vital role in product based industries. Most of the literature concentrates on quality checking of material based on dynamic motion of image capturing. This paper presents a noval approach for quality checking of wooden material based on correlation Coefficient based feature extraction technique. Pasquale Flauto and Salvatore Musella [1] has proposed a technique and quality control methods used during the development phases of an expert system prototype, known as Hydronet for conventional (procedural)software systems used by most informatics leading companies. Junfeng Li and Wenzhan Dai al.[2] has proposed the algorithm that makes full use of perfect integral comparison mechanism of the correlation coefficient and the well matching of discrete wavelet transform with multichannel model of human visual system. The algorithm can not only evaluate the integral and detail quality of image fidelity accurately but also bears more consistency with the human visual system then the traditional method PSNR. Christof Knoess et al.[3] has presented their work on daily quality checking of the natural background radioactivity from the new scintillator material LSO as a uniform source. Liwei Wang et al [4] has proposed that the matrices based 2-D algorithms are equivalent To special cases of image block based feature extraction. This method reduces the computational effort and the possibility of singularity in feature extraction Chulhee Lee and Euisun Choi [5] has proposed a method to optimize feature extraction for multiclass problems. The algorithm consistently provides better performances compared with the conventional feature extraction algorithms. Yongzhi $\mathrm{Li}$ et al [6] has proposed the method with more powerful capability to eliminate the statistical correlation between features and improve efficiency of feature extraction. This is better method than original KMMC and kernel principal component analysis (KPCA) in terms of efficiency and stability about feature extraction. Andreas Hanemann and Martin Sailer .[7] has presented a framework for the new kind of event correlation which is called service-oriented event correlation. This bridges the gap between the management of the infrastructure and the offer of services for the customers with respect to the service fault diagnosis. The organization of the paper is as follows: section 2 describes statistical features extracted from image section 3 focuses on outline of the approach, section 4 deals on experiment and results, section 5 deals with discussion and section 6 concludes with conclusion.

\section{DESCRIPTIVE FEATURES}

Today, industrial inspection systems often employ standard line or matrix cameras connected to some processing unit, typically a PC. By using smart sensor technology together with a real-time parallel processing array, we are capable of acquiring new types of images which can be processed more efficiently. The price of this type of system is expected to be significantly lower since it requires no special purpose hardware and all algorithms are implemented in software. The wood industry is one of the fields where machine vision has found practical use. There exist a number of systems, commercial as well as research for automatic wood inspection and quality sorting. The basic task for a wood inspection system is the detection of various defects, such as knots and checks. Other measuring principles, such as range imaging, must also be added to the system in order to detect all relevant properties of the wood surface. This will significantly increase the total cost of the system

The extensive set of timber images have been collected from the $U$ Oulu wood and knots database [8] for feature extraction. The typical defects of wood includes sound knots, dry knots, encased knots, decayed knot and Knot hole. All the defects are depicted in Figures.1 to 5. Other types of defects are Resin pocket, core stripe, split, and wane and Blue stain, brown stain, bark pocket, and mould are shown in Figure 6 and Figure7. Features are extracted from each block of the image and stored in separate array. Mean, median, mode, min, max, variance, covariance, standard deviation and correlation coefficient are extracted for each block of the master piece of the wood image. The same features are extracted for defective wooden 
parts. The results are compared with correlation coefficient by setting threshold value.

\section{A. Typical Defects of Wood}

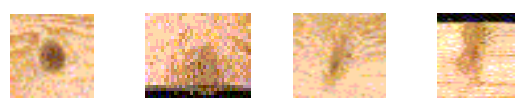

Fig.1Sound knots (normal, edge, leaf, and horn)

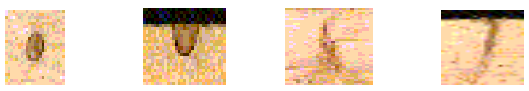

Fig. 2 Dry knots (normal, edge, leaf, and horn)

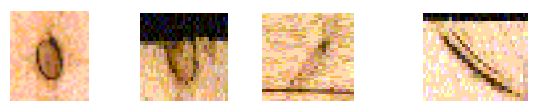

Fig. 3 Encased knots (normal, edge, leaf, and horn)
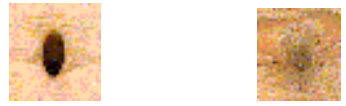

Fig. 4 Decayed knot

Fig.5 Knot hole

\section{B. Other Defects}
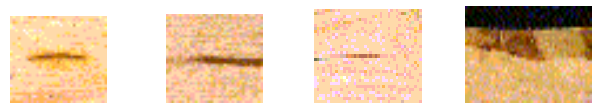

Fig. 6 Resin pocket, core stripe, split, and wane
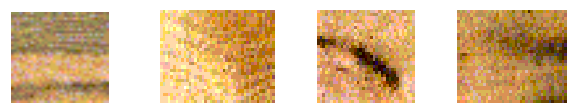

Fig. 7 Blue stain, brown stain, bark pocket, and mould

\section{OUTLINE OF THE APPROACH}

In general there is no universally applicable quality checking technique that will work for all applications. The overall complexity increases for the quality checking of a wooden material in manual processing. The proposed approach is well suitable for product based industries. The quality checking process is done in five stages.

1 Capturing the image and converting a colored image into gray scale image. 2 Split the image into16 blocks. 3 Features Mean, median, mode, min, max, variance, covariance, standard deviation are extracted for each blocks. 4 Normalise the values and calculate correlation coefficient to find the material quality accuracy. 5 If the correlation coefficient is above 0.98 ,the material is considered to be qualified else disqualified. Flowchart is given in figure 8.

Stage 1: Capture the image and convert into greyscale image.

Stage 2 : Splitting the image into 16 equal blocks with the image size (64X64) .

Stage 3: Features are extracted for each spitted block.

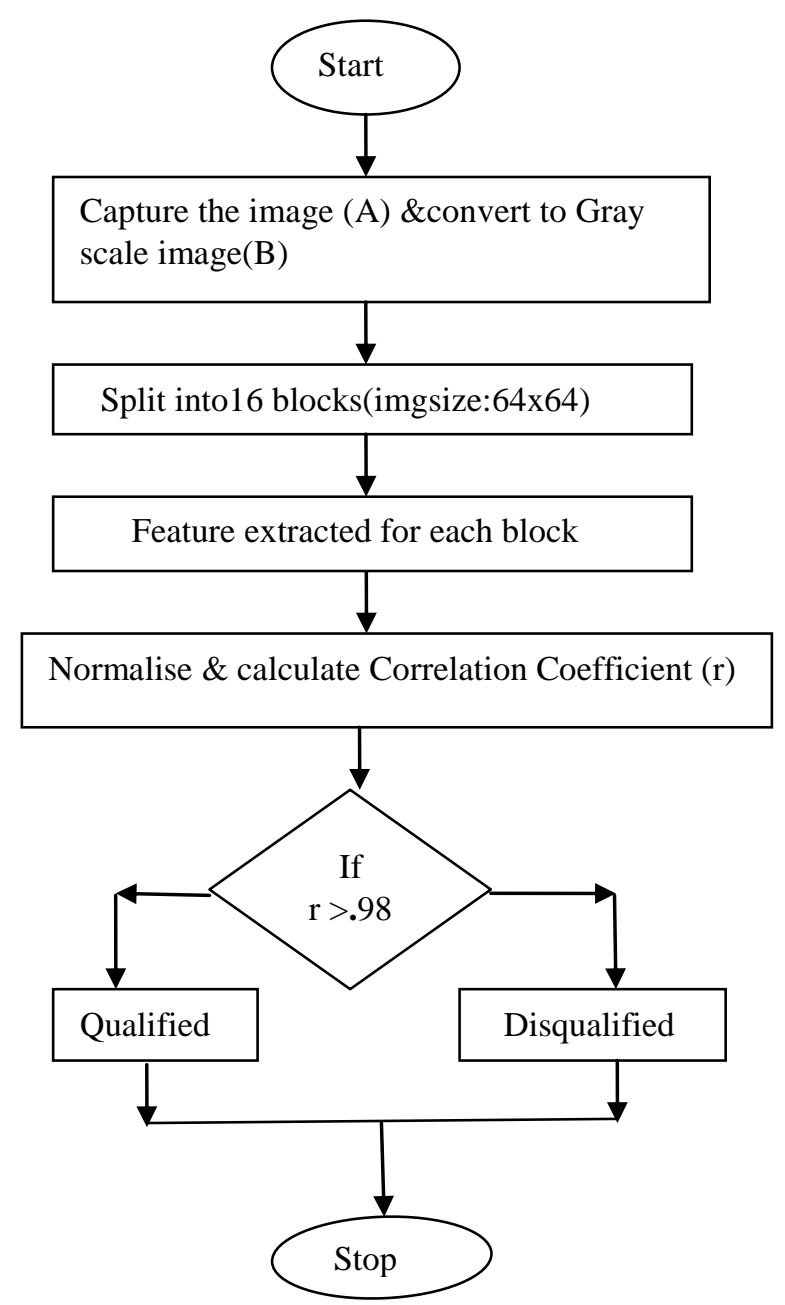

Fig. 8

Stage 4: Normalise the Extracted Features and Calculate Correlation Coefficient(r) using equation (1).

$$
\text { Norm }=\frac{x-\operatorname{mean}(x)}{\operatorname{Std}(x)}
$$

Stage 5: Check the Correlation Coefficient (r) is above 0.98 for quality of material as shown in Fig 8. else disqualified.

CorrCoeff: The matrix $\mathrm{R}=\operatorname{corrcoef}(\mathrm{X})$ is related to the covariance matrix $\mathrm{C}=\operatorname{cov}(\mathrm{X})$ using equation (2).

$$
R\left(i_{*} j\right)=\frac{c(i, j)}{\sqrt{c(i, i) c(j, j)}}
$$

\section{EXPERIMENTAL RESULTS}

The experiment is done with typical defective wooden image collected from Olu Wood and Knots database as input for checking the quality of lumber and the results are presented with few tabulated values of the images.

Stage1:The captured image is of the dimension 64X64 pixels for three different types of wood as shown in figure 8.The features are extracted per block are 8. For sixteen block there will be 128 features per image. 
TABLE 1
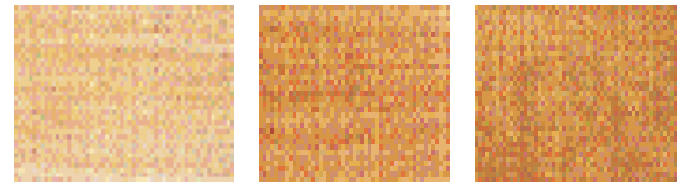

Fig. 8 Three Different types of Lumber

Stage 2: Capturing of the Mater piece Image and the Image is Splitted into 16 Equal Blocks, of size [16 X 16].

\begin{tabular}{|c|c|c|c|}
\hline 1 & 2 & 3 & 4 \\
\hline 5 & 6 & 7 & 8 \\
\hline 9 & 10 & 11 & 12 \\
\hline 13 & 14 & 15 & 16 \\
\hline
\end{tabular}

Fig. 9 Splitting of the image into 16Blocks

Stage 3: Features Mean, Standard deviation, variance, covariance are Extracted for each block. Blocks are stored in database. The results are kept in separate variables. The array editor for mean and standard deviation are shown in figure 10 and figure 11.

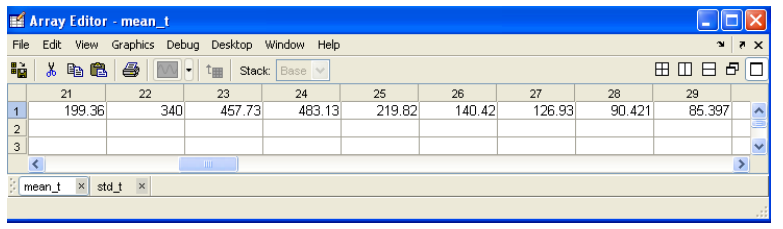

Fig. 10 Mean extracted from each block

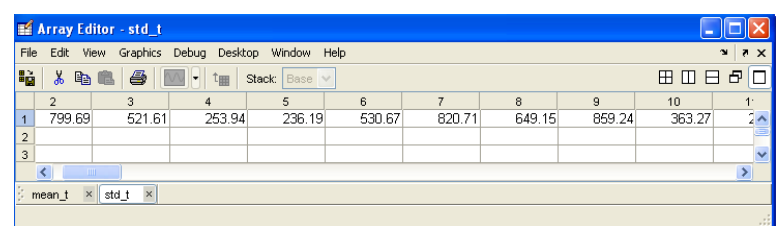

Fig. 11 Standard Deviation extracted from each block

Stage 4: Normalise the Extracted Features and Calculate Correlation Coefficient for master piece image.

Stage 5: If Correlation Coefficient (r) value calculated for each image and is presented in Table 1 . The $r$ value is above the threshold then given image matches with Master piece image and it is qualified. otherwise it is disqualified image. A sample of code for correlation coefficient is shown in figure 12 .

\section{DETECTING DEFECTS WITH CORRELATION COEFFICIENT BY SETTING THRESHOLD VALUE $(0.98)$}

\begin{tabular}{|l|c|l|l|}
\hline Master Images & $\begin{array}{c}\text { Image given } \\
\text { as Input } / \\
\text { Defective } \\
\text { Images }\end{array}$ & $\begin{array}{c}\text { Correlation } \\
\text { Coefficient } \\
\text { value }\end{array}$ & \multicolumn{1}{c|}{$\begin{array}{c}\text { Final } \\
\text { Result }\end{array}$} \\
\hline & & 0.9971 & Qualified \\
\hline & & 0.9824 & Qualified \\
\hline & & 0.9458 & Disqualified \\
\hline & & 0.8953 & Disqualified \\
\hline & & 0.962 & Disqualified \\
\hline
\end{tabular}

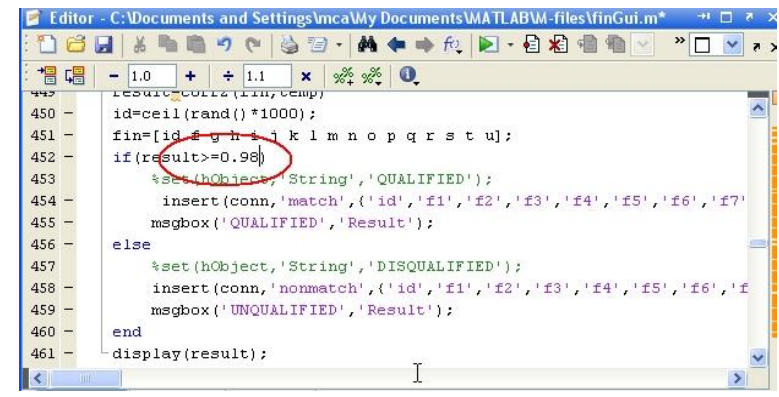

Fig. 12 Setting Threshold for Correlation Coefficient

\section{CONCLUSION}

The quality checking becomes important in all aspects of the industrial work. since product based industries focus on quality checking of a material, we have experimented with three different types of woods and the corresponding defective wood are given as input and the results are presented. Image based quality checking of a material still remains a challenging task. An attempt has been made to check the quality of material (eg. Lumber) by capturing image. The proposed work is more reliable and accurate. Our future work includes the classification of Lumber based on texture features and processing of color wooden images.

\section{REFERENCES}

[1] Pasquale Fiauto and Salvatore Musella, "Quality Control within an Expert System Prototype Development" Proc. Fourth International Conference on SoftwareEngineering and KnowledgeEngineering , pp. 435 - 442, June 1992. Digital Object Identifier 10.1109/SEKE.1992.227959

[2] Junfeng Li and Wenzhan Dai, "Quality Assessment Based on the Correlationcoefficient and the 2-D Discrete Wavelet Transform" Proc. IEEE International Conference on Automation and Logistics, pp. 789 - 793, Aug 2009. Digital Object Identifier 10.1109/ICAL.2009.5262815

[3] Christof Knoess, Tim Gremillion, Matthias Schmand, Mike E. Casey, Lars Eriksson, Mark Lenox, Jon T. Treffert,Stefan Vollmar, Guenter Fluegge, Klaus Wienhard, Wolf-Dieter 
Heiss, and Ron Nutt, “ Development of a Daily Quality Check Procedure for the High-Resolution Research Tomograph (HRRT) Using Natural LSO Background Radioactivity" Proc. IEEE Transactions on vol. 36, Issue 1, pp. 194 - 197,Feb 2006. Digital Object Identifier 10.1109/SEKE.1992.227959

[4] Liwei Wang, Xiao Wang and Jufu Feng, "Correspondence On Image Matrix Based Feature Extraction Algorithms," Proc. IEEE Transactions on Systems, Man, and Cybernetics, Part B vol. 39, Issue 3, pp.521 - 528,Mar 2001. Digital Object Identifier 10.1109/TSMCB.2005.852471

[5] Chulhee Lee and Euisun Choi, "Optimizing Feature Extraction for Multiclass Problems,"Proc. 15th International Conference on Pattern Recognition vol. 2, pp.402 405,Sept 2000. Digital Object Identifier 10.1109/ICPR.2000.906097
[6] Yong-zhi Li, Feng Ming, Jing-yu Yang and Ren-Liang Pan, "An Efficient Method of Nonlinear Feature Extraction Based on SVM," Proc. 9th International Conference on Control, Automation, Robotics and Vision,ICARCV '06.pp.1 - 6,Dec $2006 . \quad$ Digital Object Identifier 10.1109/ICARCV.2006.345461

[7] Andreas Hanemann and Martin Sailer, "A Framework for Service Quality Assuranceusing Event Correlation Techniques" Proc. $\quad \sum$ Advanced Industrial Conference on Telecommunications/Service Assurance with Partial and Intermittent Resources Conference/ E-Learning on Telecommunications Workshop, pp.428 - 433,July 2005. Digital Object Identifier 10.1109/AICT.2005.7

[8] U Oulu wood and knots database [Online]. Available: http://www.ee.oulu.fi/ olli/Projects/Lumber.Grading.html 